\title{
Influence of Ultraviolet Radiation on Surface Electric Potential of P(VDF-TrFE) Films
}

\author{
EDGARS LुAKSA, ${ }^{1}$ ALEXEI KATASHEV,${ }^{1}$ YURI DEKHTYAR, ${ }^{1}$ \\ SERGEY G. YUDIN, ${ }^{2}$ AND MARINA ROMANOVA ${ }^{1, *}$ \\ ${ }^{1}$ Institute of Biomedical Engineering and Nanotechnology, Riga Technical \\ University, Riga, Latvia \\ ${ }^{2}$ A.V. Shubnikov Institute of Crystallography of the Russian Academy \\ of Sciences, Moscow, Russia
}

\begin{abstract}
Changes in surface electric potential of $P(V D F-T r F E ~ 70: 30)$ films deposited on glass substrate under influence of $U V$ radiation were investigated. It was found that after 6 minutes of UV irradiation surface electric potential of the films decreased and no changes were observed further increasing irradiation time. Surface electric potential exponentially relaxed to its initial value within 30 min after the irradiation was stopped. The study suggests that P(VDF-TrFE 70:30) films deposited on glass substrate are sensitive to UV radiation and might be used as a material for an UV detector, however, further studies are required.
\end{abstract}

Keywords P(VDF-TrFE); thin films; surface electric potential; ultraviolet radiation; dosimetry; Kelvin force microscopy

\section{Introduction}

Poly(vinylidene fluoride-trifluoroethylene) $[\mathrm{P}(\mathrm{VDF}-\mathrm{TrFE})]$ is a polymer well-known for its ferroelectric properties [1]. In its piezoelectric form, $\mathrm{P}(\mathrm{VDF}-\mathrm{TrFE})$ finds use in transducer devices where mechanical energy is converted into electrical energy and vice versa. Such polymer can be used in a variety of sensors and actuators such as artificial muscles and organs, medical imaging, blood-flow monitors, microphones, smart skins, underwater acoustic transducers, seismic monitors, fluid pumps and valves, surface acoustic wave devices, robots, and tactile sensing devices $[1,2]$. Ability of P(VDF-TrFE) to change its polarization upon application of an external electric field is used in nonvolatile memory and data-storage devices [3].

The target of the research was to examine application of the P(VDF-TrFE) films consisting of $70 \%$ vinylidene fluoride (VDF) and $30 \%$ trifluoroethylene (TrFE), or P(VDFTrFE 70:30), as possible detector of ultraviolet (UV) radiation. P(VDF-TrFE 70:30) films were deposited on glass substrate using Langmuir-Blodgett technique. Size of the samples was $1 \times 1 \mathrm{~cm}$. The films were fabricated in Shubnikov Institute of Crystallography of the Russian Academy of Sciences in collaboration with Sergey G. Yudin, leading researcher. 


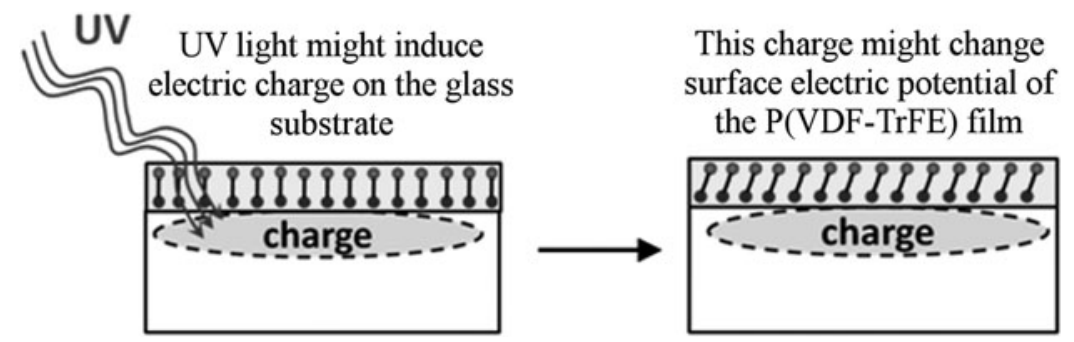

Figure 1. The hypothesis was proposed that electric charge was created on surface of the glass substrate due to absorption of UV radiation. This charge might change polarization and surface electric potential of the $\mathrm{P}(\mathrm{VDF}-\mathrm{TrFE})$ film.

\section{Methods}

The P(VDF-TrFE 70:30) films were UV irradiated with HAMAMATSU PHOTONICS Lightningcure 5 series xenon-mercury lamp L8222-01 (250-400 nm). The films were irradiated at distance $40 \mathrm{~cm}$ between the film surface and output of optical fiber of the UV lamp. This distance was chosen to avoid heating of the films. According to the Hamamatsu Photonics datasheet, intensity of UV radiation at this distance was about $250 \mathrm{~mW} / \mathrm{cm}^{2}$.

It was hypothesized that UV radiation can be absorbed by the glass substrate and can induce electric charge on its surface. Amount of the induced charge might depend on irradiation time. The induced electric charge in turn might change polarization of the P(VDF-TrFE 70:30) film (Fig. 1). These polarization changes might influence surface electric potential of the film. Surface electric potential of the films before and after irradiation was measured using Kelvin force microscopy. Measurements were performed using atomic force microscope (AFM) Solver P-47 PRO. To measure surface electric potential of the same area of the film before and after irradiation, UV irradiation was always performed inside AFM. Glass substrate without P(VDF-TrFE 70:30) film was also irradiated and its surface electric potential was measured.
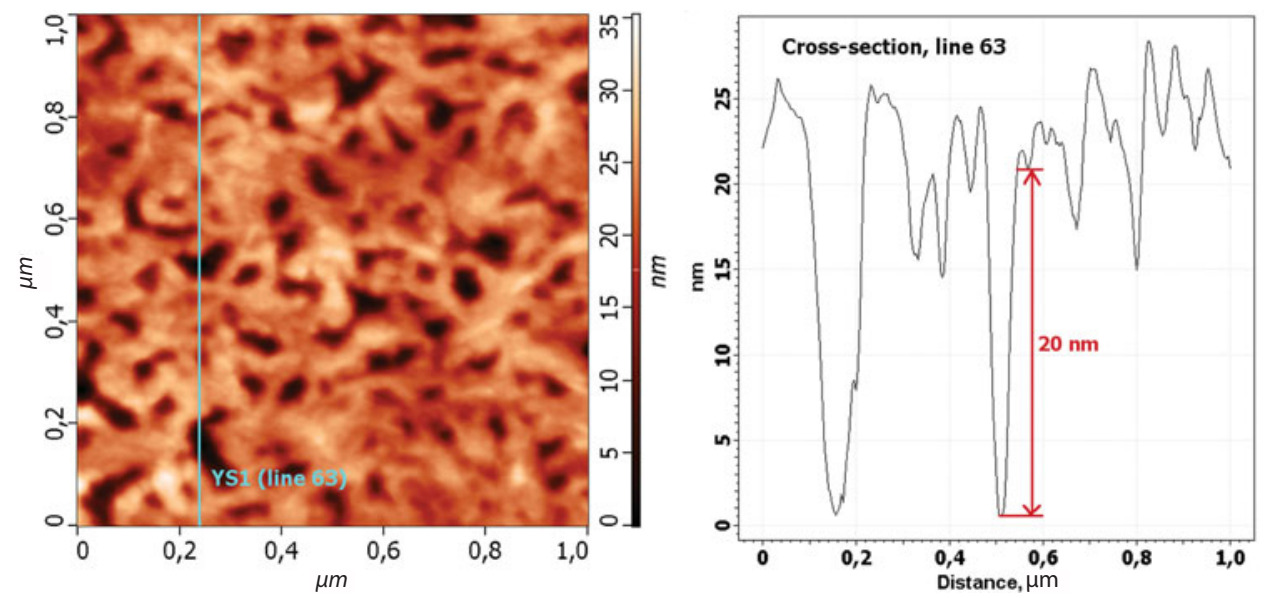

Figure 2. AFM topography of $10 \mathrm{ML}$ thick P(VDF-TrFE 70:30) film. Scan size $10 \times 10 \mu \mathrm{m}$. Color gradation on the vertical Z-axis is from $0 \mathrm{~nm}$ (dark regions) to $35 \mathrm{~nm}$ (light regions). 

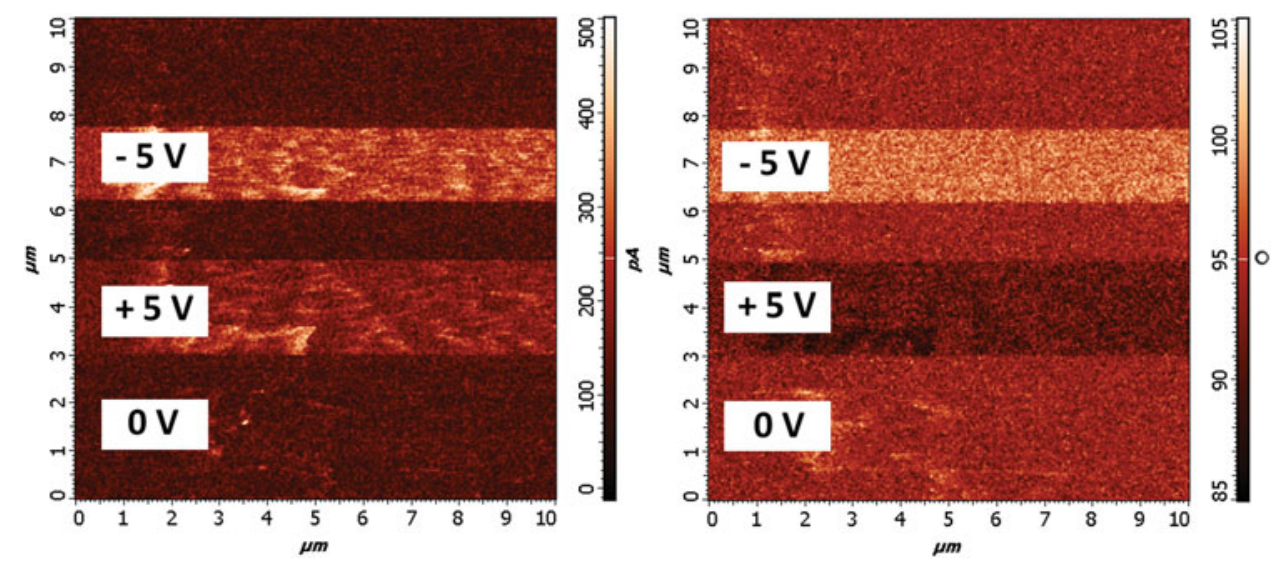

Figure 3. Piezoresponse Z-amplitude (on the left) and piezoresponse phase (on the right) images of the 11 ML P(VDF-TrFE 70:30) film. DC bias $+5 \mathrm{~V}$ and $-5 \mathrm{~V}$ was applied during scanning (Color figure available online).

\section{Results}

AFM topography of the 10 monolayer (ML) thick P(VDF-TrFE 70:30) films deposited on glass substrate is shown in Fig. 2.

To test ferroelectric properties of the films, piezoresponse force microscopy mode of AFM was used. DC bias voltage $+5 \mathrm{~V}$ and $-5 \mathrm{~V}$ was applied to the $11 \mathrm{ML}$ thick P(VDFTrFE 70:30) film during scanning. The applied DC bias voltage changed piezoresponse Z-amplitude and piezoresponse phase of the film (Fig. 3). Piezoresponse phase image clearly identifies regions with different polarization states which have resulted from the opposite polarity of the applied bias voltage. The external DC bias voltage applied in this experiment was used to model electric charge induced on surface of the glass substrate during UV irradiation (Fig. 1). This experiment proved that external electric field changes polarization of the examined $\mathrm{P}(\mathrm{VDF}-\mathrm{TrFE} 70: 30)$ films.

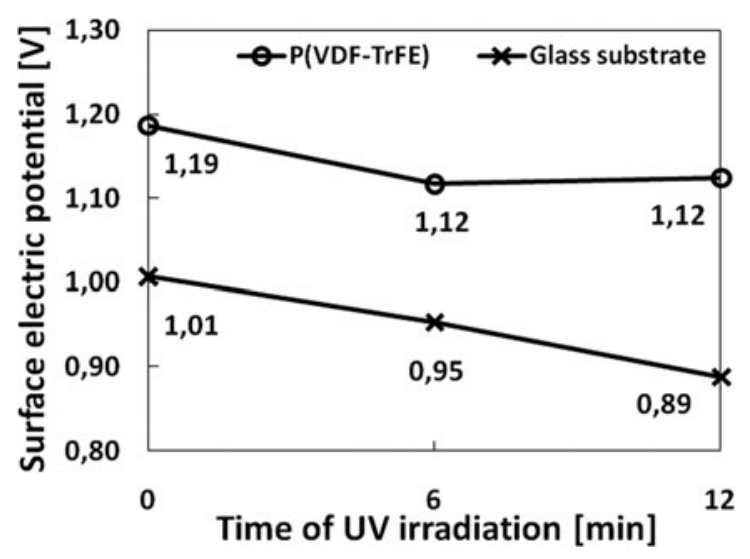

Figure 4. Surface electric potential of $10 \mathrm{ML}$ thick P(VDF-TrFE 70:30) film and the glass substrate after 0,6 and 12 minutes of UV irradiation. 


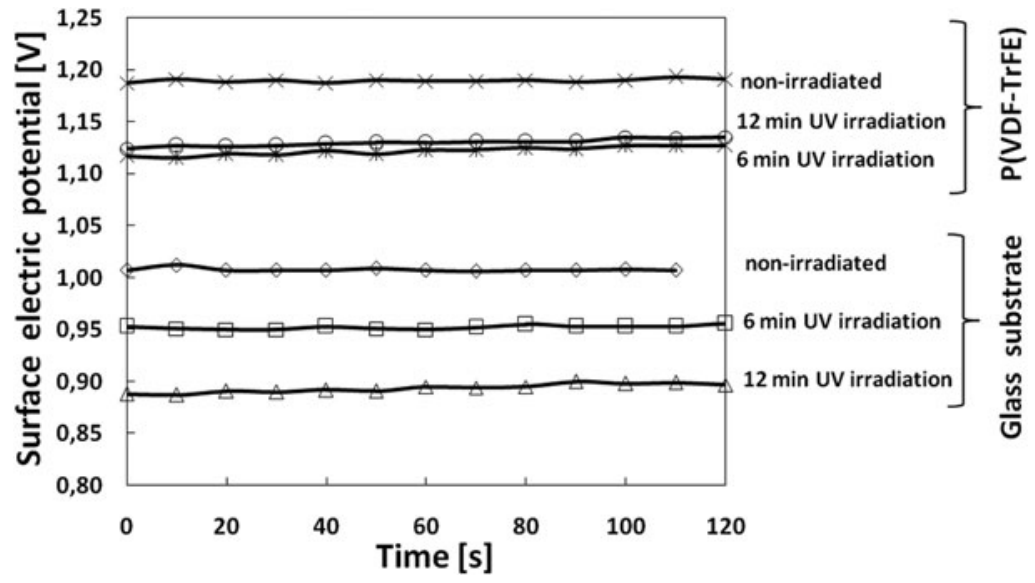

Figure 5. Stability of surface electric potential of 10 ML thick P(VDF-TrFE 70:30) film and the glass substrate during first two minutes after UV irradiation was turned off.

Figure 4 shows changes of surface electric potential of 10 ML thick P(VDF-TrFE 70:30) films and of the glass substrate after 0, 6 and 12 minutes of UV irradiation. Surface electric potential of the P(VDF-TrFE 70:30) films decreased after 6 minutes of UV irradiation and did not change when irradiation time was increased. Surface electric potential of the glass substrate also decreased after 6 minutes of UV irradiation, however, unlike the film, surface electric potential of the glass substrate continued to decrease when irradiation time was increased.

Explanation was proposed that despite the fact that UV radiation continued to change electric charge on the glass substrate surface when irradiation time exceeded 6 minutes, these changes had no further effect on polarization of the P(VDF-TrFE 70:30) film (see hypothesis in Fig. 1), which at that time already had arrived to saturation.

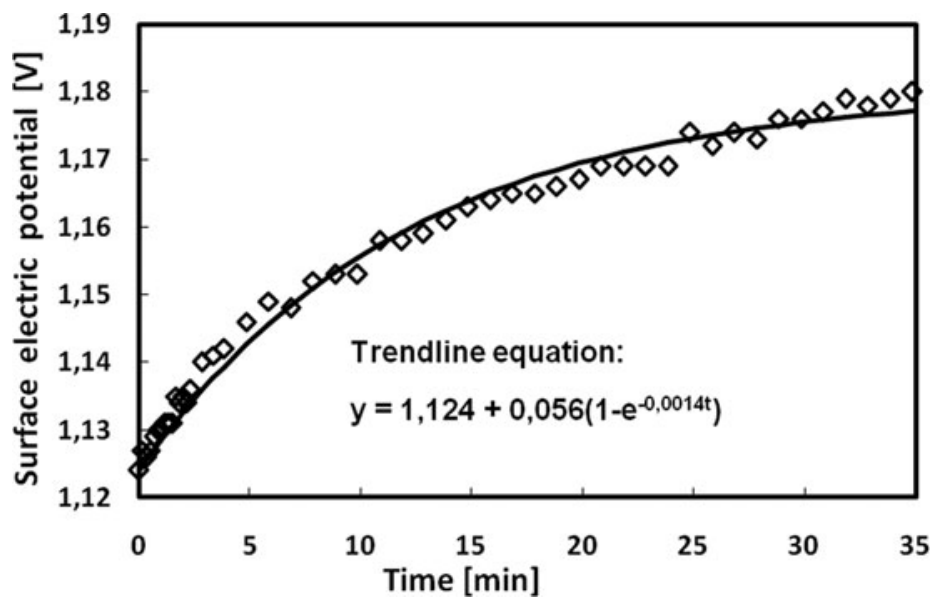

Figure 6. Long-term relaxation of surface electric potential of 10 ML thick P(VDF-TrFE 70:30) film to its initial value after UV radiation was turned off. The film was irradiated with UV during 12 minutes. 
In order to examine stability of surface electric potential after UV irradiation, the P(VDF-TrFE 70:30) films kept being measured with intervals 10 seconds after UV light was turned off (Fig. 5, time on the horizontal axis). The measurements were performed up to 120 seconds and demonstrated that surface electric potential was stable during this period of time.

Long-term stability of surface electric potential of 10ML thick P(VDF-TrFE 70:30) films after UV radiation was turned off was also studied (Fig. 6). The films were irradiated with UV during 12 minutes. Surface electric potential was measured with 1 minute interval after turning off the source of UV radiation. It was found that surface electric potential returned to the initial value present before the UV irradiation. Relaxation of the potential happened within half an hour and obeyed the exponential law (see the Trendline equation in Fig. 6).

\section{Conclusions}

Kelvin force microscopy evidence that UV radiation decreases surface electric potential of Langmuir-Blodgett P(VDF-TrFE 70:30) films deposited on glass substrate. Presumably, this is because UV radiation is absorbed by surface of the glass substrate, changing its surface electric charge, which, in turn, influences the P(VDF-TrFE 70:30) film changing its polarization. Surface electric potential of the film stabilizes after 6 minutes of UV radiation and does not change with further increase of irradiation time. However, surface electric potential of the glass substrate continues to decrease when irradiation time is increased. After UV radiation is turned off, surface electric potential of the P(VDF-TrFE 70:30) films relaxes to the initial value which is present before the irradiation. Relaxation happens within half an hour and obeys exponential law.

Our studies suggest that Langmuir-Blodgett P(VDF-TrFE 70:30) films deposited on the glass substrate are sensitive to UV radiation. However, further investigation is required to examine possibility to use these films as a detector of UV radiation.

\section{Acknowledgment}

Authors would like to thank team of Mr. Sergey G. Yudin, the leading researcher of Shubnikov Institute of Crystallography of the Russian Academy of Sciences, who provided $\mathrm{P}(\mathrm{VDF}-\mathrm{TrFE}$ 70:30) films for the research.

\section{References}

1. H. S. Nalwa, Ferroelectric Polymers: Chemistry, Physics and Applications. New York: Marcel Dekker; (1995).

2. Q. M. Zhang, V. Bharti, G. Kavarnos, and M. Schwartz (Ed.), Poly(Vinylidene Fluoride) (PVDF) and its Copolymers. In: Encyclopedia of Smart Materials, vol. 1-2. New York: John Wiley \& Sons; 807-825 (2002).

3. S. Ducharme, T. J. Reece, C. M. Othon, and R. K. Rannow, Ferroelectric Polymer LangmuirBlodgett Films for Nonvolatile Memory Applications. IEEE Transactions on Device and Material Reliability. 5, 720-735 (2005). 